\title{
NONLINEAR \\ CONTROL SYSTEMS
}

Robert Lien Cosgriff, Ph.D.

Assistant Professor of Electrical Engineering

Associate Supervisor of Antenna Laboratory

The Ohio State University

McGRAW-HILL BOOK COMPANY, INC.

New York Toronto London

1958 